\title{
Modelling and Simulation of the Coupled Rigid-flexible Multibody Systems in MWorks
}

\author{
Xie Gang ${ }^{1}$, Zhao Yan ${ }^{1}$, Zhou Fanli* ${ }^{2}$, Chen Liping ${ }^{1}$ \\ ${ }^{1}$ CAD Center, Huazhong University of Science and Technology, Wuhan, China, 430074 \\ ${ }^{2}$ Suzhou Tongyuan Software \& Control Tech. Co. Ltd, Suzhou, China, 215123 \\ $\{$ xieg, zhaoy, zhoufl, chenlp $\} @$ tongyuan.cc
}

\begin{abstract}
Aiming to the design challenge of modern mechatronic products, this paper presents a method to simulate the coupled rigid-flexible system in MWorks. Firstly, the component mode synthesis (CMS) technique is introduced and the Craig-Bampton method is adopted to build the flexible-body model. The general flexible-body model named FlexibleBody is developed based on the standard MultiBody library in Modelica, which describes the small and linear deformation behavior (relative to a local reference frame) of a flexible-body that undergoes large and non-linear global motion. In the model, the modal neutral file (MNF) is introduced as a standard interface to describe the constraint modes. Secondly, the model is used to construct a library of boom system of concrete pump truck and the simulations covering the expanding and folding process are carried out based on both the rigid multibody and the coupled rigid-flexible system models. Finally, the influence to dynamics performance of the boom system is analyzed and the conclusion is drawn. The method in this paper provides an effective approach to build unified model and simulate flexible-body in multidomain engineering systems.
\end{abstract}

Keywords: rigid-flexible system; concrete pump truck; MWorks

\section{Introduction}

Much industrial equipment is mechatronic and contains high-speed, lightweight, and high-precision mechanical system. In these mechanical systems one or more structural components often need to consider the deformation effects for design analyses. The integrated design and simulation of the mechatronic systems with flexible bodies make the multidisciplinary challenge. When designing such a mechatronic system, the performance requirements must be satisfied and the strength of the system must be guaran- teed. Therefore, stress and deformation of machine components have to be predicted in the design process.

The increasing computational power of current computer enables to model a multibody system as 3D deformable body using the finite element method. The flexible multibody dynamics is the subject concerned with the modeling and analysis of constrained deformable bodies that undergo large displacements and rotations. DLR ${ }^{\circledR}$ FlexibleBodies Library ${ }^{[1,2]}$ provides the general flexible model so that users can simulate the elastic deformation of flexible-body in a modal synthesis way, in which the standard input data (SID) ${ }^{[3,4]}$ file should be offered by a third-party software. In SID file, Guyan reduction and Ritz approximation are adopted. The Rayleigh-Ritz method ${ }^{[4]}$ chooses an approximate form for the eigenfunction with the lowest eigenvalue. In the Guyan reduction method ${ }^{[4]}$, a set of user-defined master nodes are retained and the remaining set of slave nodes are removed by condensation. Only stiffness properties are considered during the condensation, and inertia coupling of master and slave nodes are ignored. Based on an improved Craig-Bampton method ${ }^{[5-8]}$, MSC.ADAMS ${ }^{\circledR}$ adopts the modal neutral file ${ }^{[9]}$, which can be exported by some finite element software, to drive the animation of the flexible body. The MNF is a binary file that contains the location of nodes and node connectivity, nodal mass and inertia, mode shapes, generalized mass and stiffness for mode shapes. The mode shapes in modal neutral file, which contain the interface constraint modes, are revised effectively after modal truncation. So the Craig-Bampton method is more accurate and has been widely used in engineering ${ }^{[10-14]}$. But the software, just like ADAMS, mainly focuses on modeling and simulation of the pure mechanical system and lacks support of multi-domain physical systems. In order to model and simulate the mechatronic products composed of mechanical, electronic, hydraulic, and control engineering systems, the co-simulation 
should be performed with other software such as Matlab/Simulink ${ }^{\circledR}$, LMS.AMESim $^{\circledR}$, etc.

In this paper, the FlexibleBody model is developed based on the component mode synthesis (CMS) and the improved Craig-Bampton method ${ }^{[9,11]}$. An external $\mathrm{C}$ function MNFParser is programmed to get the mode shapes data in the model. The finite element analysis (FEA) results can then be incorporated into a part model by superimposing the flexible-body deflection on the motion of rigid-body. The postprocessor tool in MWorks ${ }^{[15]}$ is also improved to support the nephogram animation of the deformation. As an example, a boom system library of the concrete pump truck is developed. And the simulations covering the expanding and folding process are carried out based on both the rigid multibody and the coupled rigid-flexible system models. The simulation results are compared and it shows the coupled rigid-flexible system is more conformable with the actual boom system.

\section{The Flexible-Body Model}

To build the model of flexible-body based on CMS the mode shapes data in MNF is provided by thirdparty finite element analysis (FEM) software. So the MNF file needs to be parsed. Then the equations of coupled elastic deformation and rigid body are established. To animate the deformation of flexible-body, the postprocessor needs to have the ability to show nephograms. The whole process in MWorks is shown in Figure 1.

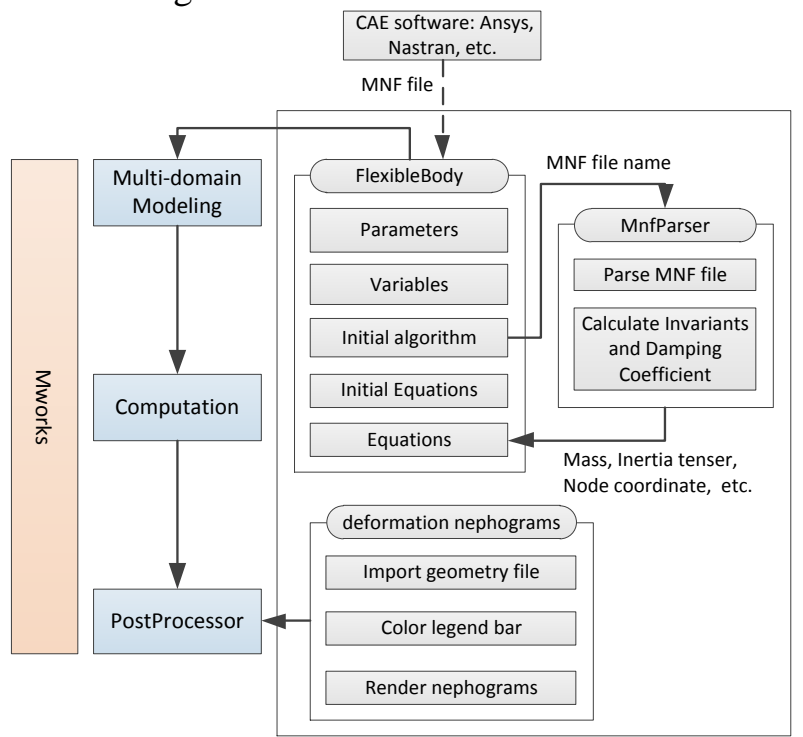

Figure 1: Development process of flexible-body in MWorks

\subsection{Theoretical Background}

In this paper, only small, linear body deformations relative to a local reference frame are considered, while that local reference frame indicates large, nonlinear global motion.

The discretization of a flexible component into a finite element model represents the infinite number of degree of freedom (DOF) with a finite element. The basic premise of modal superposition is that the deformation behavior of a component with a very large number of nodal DOF can be captured with a much smaller number of modal DOF. This reduction in DOF is called modal truncation.

The linear deformations of the nodes of this finite element mode, u, can be approximated as a linear combination of a smaller number of translational mode shapes matrix ${ }^{[9]}, \boldsymbol{\Phi}_{T}=\left(\phi_{T 1}, \phi_{T 2}, \cdots, \phi_{T M}\right)$.

$$
\mathbf{u}=\mathbf{\Phi}_{T} \mathbf{q}+\overline{\mathbf{\Phi}}_{T} \overline{\mathbf{q}} \approx \phi_{T 1} q_{1}+\cdots+\phi_{T M} q_{M}=\sum_{i=1}^{M} \phi_{T i} q_{i}
$$

Where $\overline{\boldsymbol{\Phi}}_{T}$ and $\overline{\mathbf{q}}$ are the truncated mode shapes matrix and the truncated modal coordinates respectively. $M$ is the number of mode shape, and $\mathbf{q}=\left(q_{1}, q_{2}, \cdots, q_{M}\right)^{T}$ is the modal coordinates.

Similar to equation(1), as the body deforms, every node rotates through small angles relative to its reference frame. These angles are obtained using a modal superposition ${ }^{[9]}$.

$$
\boldsymbol{\theta}=\boldsymbol{\Phi}_{R} \mathbf{q}+\overline{\boldsymbol{\Phi}}_{R} \overline{\mathbf{q}} \approx \sum_{i=1}^{M} \phi_{R i} q_{i}
$$

Where $\boldsymbol{\Phi}_{R}=\left(\phi_{R 1}, \phi_{R 2}, \cdots \phi_{R M}\right)$ is the slice of rotational mode shapes matrix.

In the studies referring to $[3,4]$, the eigenvectors of an unconstrained system be used. Eigenvectors are found to provide an inadequate basis in system level modeling. To improve the accuracy of the system model, the CMS ${ }^{[3]}$ techniques are adopted, the most general methodology is Craig-Bampton method ${ }^{[5]}$.

The Craig-Bampton method ${ }^{[5,6]}$ allows the user to select a subset of DOF which are not to be subject to modal superposition. These boundaries DOFs (or attachment DOFs) are preserved exactly in the Craig-Bampton modal basis. There is no loss in resolution of these DOF when higher order modes are truncated ${ }^{[7,8]}$.

The system DOF in Craig-Bampton method are partitioned into boundary DOF, $\mathbf{u}_{B}$, and interior DOF, $\mathbf{u}_{I}$. Two sets of mode shapes are defined, as follows [9]:

Constraint modes: These modes are static shapes obtained by giving each boundary DOF a unit displacement while holding all other boundary DOF 
fixed. The basis of constraint modes completely spans all possible motions of the boundary DOFs, with a one-to-one correspondence between the modal coordinates of the constraint modes and the displacement in the corresponding boundary DOF, $\mathbf{q}_{C}=\mathbf{u}_{B}$.

Fixed-boundary normal modes: These modes are obtained by fixing the boundary DOF and computing an eigensolution. There are as many fixed-boundary normal modes as the user desires. These modes define the modal expansion of the interior DOF. The quality of this modal expansion is proportional to the number of modes retained by the user.

The relationship between the physical DOF and the Craig-Bampton modes and their modal coordinates is illustrated by the following equation.

$$
\mathbf{u}=\left\{\begin{array}{l}
\mathbf{u}_{B} \\
\mathbf{u}_{I}
\end{array}\right\}=\left[\begin{array}{cc}
\mathbf{I} & \mathbf{0} \\
\boldsymbol{\Phi}_{I C} & \boldsymbol{\Phi}_{I N}
\end{array}\right]\left\{\begin{array}{l}
\mathbf{q}_{C} \\
\mathbf{q}_{N}
\end{array}\right\}
$$

Where $\mathbf{I}, \mathbf{0}$ are identity and zeros matrices, respectively. $\boldsymbol{\Phi}_{I C}$ is the physical displacements of the interior DOF in the constraint modes. $\boldsymbol{\Phi}_{I N}$ is the physical displacements of the interior DOF in the normal modes. $\mathbf{q}_{C}$ is the modal coordinates of the constraint modes. $\mathbf{q}_{N}$ is the modal coordinates of the fixedboundary normal modes.

The generalized stiffness and mass matrices corresponding to the Craig-Bampton modal basis are obtained via a modal transformation.

\subsection{FlexibleBody Model}

The governing differential equation of flexible-body $[9,15]$, in terms of the generalized coordinates is:

$$
\mathbf{M} \ddot{\boldsymbol{\xi}}+\dot{\mathbf{M}} \dot{\boldsymbol{\xi}}-\frac{1}{2}\left[\frac{\partial \mathbf{M}}{\partial \boldsymbol{\xi}} \dot{\boldsymbol{\xi}}\right]^{T} \dot{\boldsymbol{\xi}}+\mathbf{K} \boldsymbol{\xi}+\mathbf{f}_{g}+\mathbf{D} \dot{\boldsymbol{\xi}}+\left[\frac{\partial \boldsymbol{\psi}}{\partial \boldsymbol{\xi}}\right]^{T} \boldsymbol{\lambda}=\mathbf{Q}
$$

Where,

$\xi, \dot{\xi}, \ddot{\xi}$ are the generalized coordinates of the flexiblebody and their time derivatives.

$$
\xi=\left(\begin{array}{lllllll}
x & y & z & \psi & \varphi & \phi & q_{i,(i=1, \ldots, M)}
\end{array}\right)^{T}=\left(\begin{array}{lll}
\mathbf{x} & \boldsymbol{\psi} & \mathbf{q}
\end{array}\right)^{T}
$$

$\mathbf{M}$ is the mass matrix.

$\mathbf{K}$ is the generalized stiffness matrix.

$\mathbf{f}_{g}$ is the generalized gravitational force.

D is the modal damping matrix.

$\boldsymbol{\Psi}$ is the algebraic constraint equations.

$\lambda$ is the Lagrange multipliers for the constraints.

$\mathbf{Q}$ is the generalized applied forces.

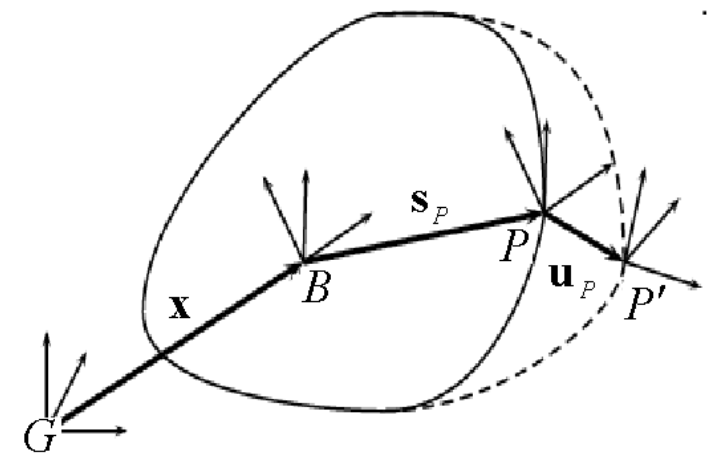

Figure 2: The position vector to a deformed point $P^{\prime}$ on a flexible body

The instantaneous location of a point that is attached to a node, $P$, on a flexible body, $B$, is the sum of three vectors, showing in Figure 2.

$$
\mathbf{r}_{p}=\mathbf{x}+\mathbf{s}_{p}+\mathbf{u}_{p}
$$

Where

$\mathbf{x}$ is the position vector from the origin of the ground reference frame to the origin of the local body reference frame of the flexible body.

$\mathbf{s}_{p}$ is the position vector of the undeformed position of point $P$ with respect to the local body reference frame of body $B$.

$\mathbf{u}_{p}$ is the translational deformation vector of point $P$, the position vector from the point's undeformed position to its deformed position. It is also expressed in the local body coordinate system. The deformation vector is a modal superposition, $\mathbf{u}_{P}=\boldsymbol{\Phi}_{T P} \mathbf{q}$. Where $\boldsymbol{\Phi}_{T P}$ is the slice from the modal matrix that corresponds to the translational DOF of node $P$.

The general flexible-body model based on CMS is developed according to the following processes.

(1) Defining the parameters: MNF file name, mass, inertia, mode shape, set of selected mode, etc.

(2) Setting the variants and default values: modal coordinates and first-order derivate, secondorder derivate, velocity, acceleration, etc.

(3) Configuring the initial algorithm: Call MNFParser function (refer to 2.3 MNF File Parser) to get the mass, inertia, mode shape, stiffness matrix, invariants, damping coefficient, etc.

(4) Setting the initial equations: just like the equations in Body model in Multibody library.

(5) Describing the equations: Force and torque balance equation, and equation (4) are defined. With modal coordinates, the deformation of the flexible-body equation (1), (2), (4) and (5).

This approach consists of the Body model in MultiBody library. The general FlexibleBody model is shown in Figure 3. 


\section{\%name}

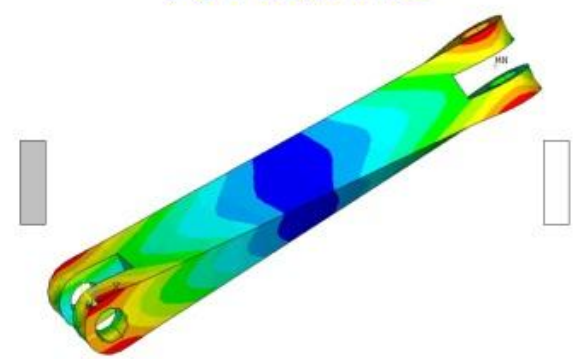

Figure 3: Icon of the FlexibleBody model

The FlexibleBody model encapsulates the complexity of details into a black box that we can use modularly without considering the detailed implementation at the top level.

\subsection{MNF File Parser}

The mode shapes data are needed to build the flexible-body model with modal synthesis method, and the MNF is adopted to express these data, which is generated by CAE software such as ANSYS ${ }^{\circledR}$, NASTRAN ${ }^{\circledR}$ etc.

Modal neutral file is a platform-independent binary file. The information in a MNF includes geometry (locations of nodes and node connectivity), nodal mass and inertia, mode shapes, generalized mass and stiffness for mode shapes, which is listed in Table 1.

Table 1: Information in MNF

\begin{tabular}{c|l}
\hline Block & \multicolumn{1}{c}{ information } \\
\hline Header & $\begin{array}{l}\text { date, program name and version, title, } \\
\text { MNF version, units }\end{array}$ \\
\hline Body properties & $\begin{array}{l}\text { mass, moments of inertia, center of } \\
\text { mass }\end{array}$ \\
\hline Interface points & Reduced stiffness and mass matrices \\
\hline Interface modes & User requests the number of modes \\
\hline Constraint modes & Interface constraint modes \\
\hline
\end{tabular}

According to its data structure, an external $\mathrm{C}$ function "MNFParser" is programed to parse the MNF file and to obtain the quality, inertia tensor, eigenvalues, modal shapes matrix, etc.

To reduce the simulation time, the nine inertia invariants ${ }^{[9]}$ are calculated beforehand from the $N$ nodes of the finite element model based on each node's mass, undeformed location coordinates in the component modes.

And the default damping coefficients are calculated according to the modal frequency.

- $1 \%$ damping for all modes with frequency lower than 100.

- $10 \%$ damping for modes with frequency in the 100-1000 range.
- $100 \%$ critical damping for modes with frequency above 1000.

The MNFParser also provides nodes coordinates and element faces of the FE model to the postprocessor for rendering deformation nephograms.

\section{Modeling and Simulation of the Coupled Rigid-flexible Boom Sys- tem}

The concrete pump truck has become a kind of indispensable machinery equipment in construction industry. It pumps concrete continuously sent by concrete mixer truck to the pouring site. The boom system is generally composed of mechanical, hydraulic and control subsystems, and the multidomain modeling and simulation is necessary for the design and validation of this system. The modeling and simulation process of boom system in MWorks is shown in Figure 4.

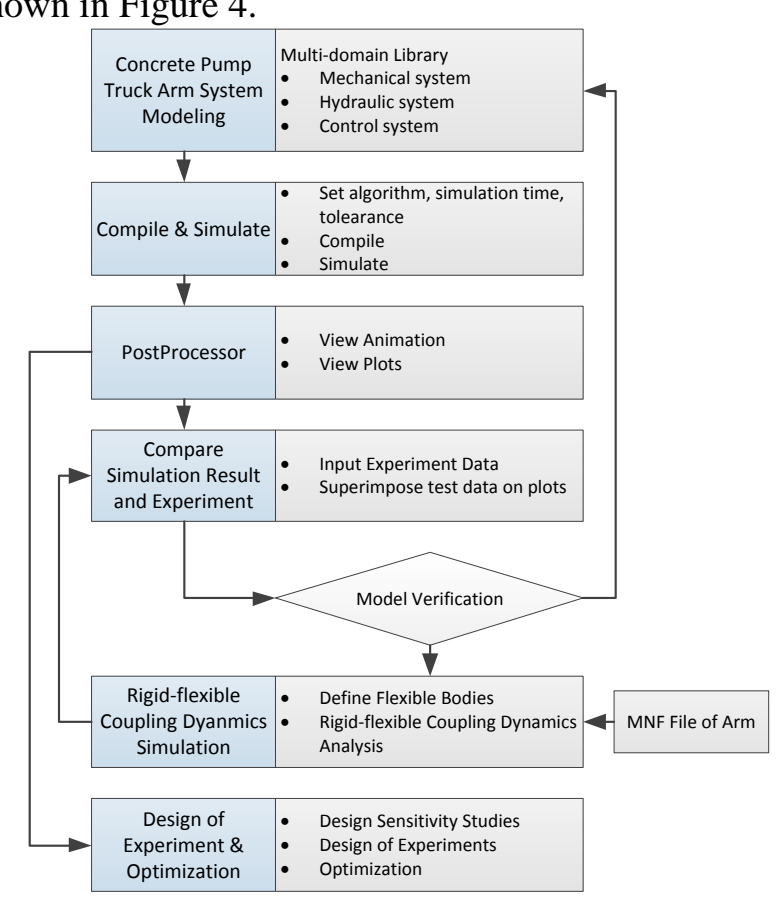

Figure 4: The modeling and simulation workflow of booms system of concrete pump truck

\subsection{Library of Boom System}

The hierarchical multi-domain library of boom system is developed based on the standard Modelica library and the hydraulic library developed by Suzhou Tongyuan Software \& Control Technology Company. Its structure is shown in Figure 5. 


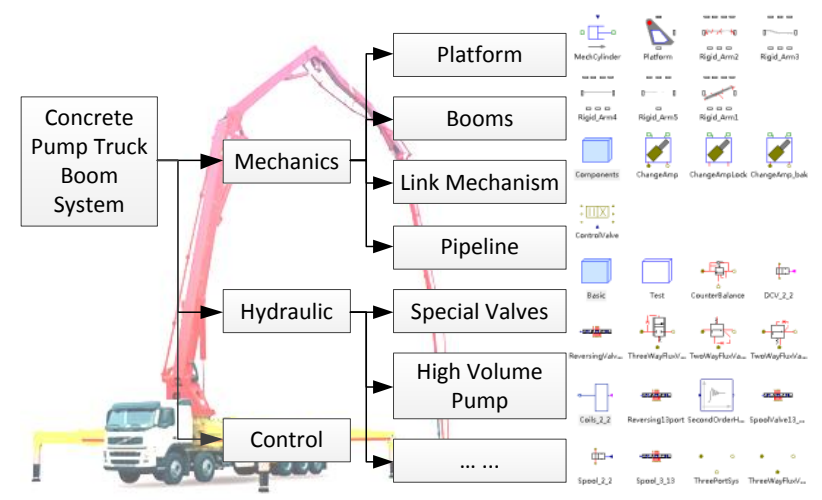

Figure 5: Structure of the booms library

The boom system library includes mechanics, hydraulic, and control subsystem library. The boom system model can be conveniently constructed by dragging and dropping based on the library.

\subsection{Simulation of Rigid Boom System}

The booms of concrete pump truck must expand and fold regularly while working. The working loads should be analyzed to ensure the safety. The control system should also be designed to satisfy the casting needs.

The hierarchical structure of the boom system model is established based on the MultiBody library, shown in Figure 6.

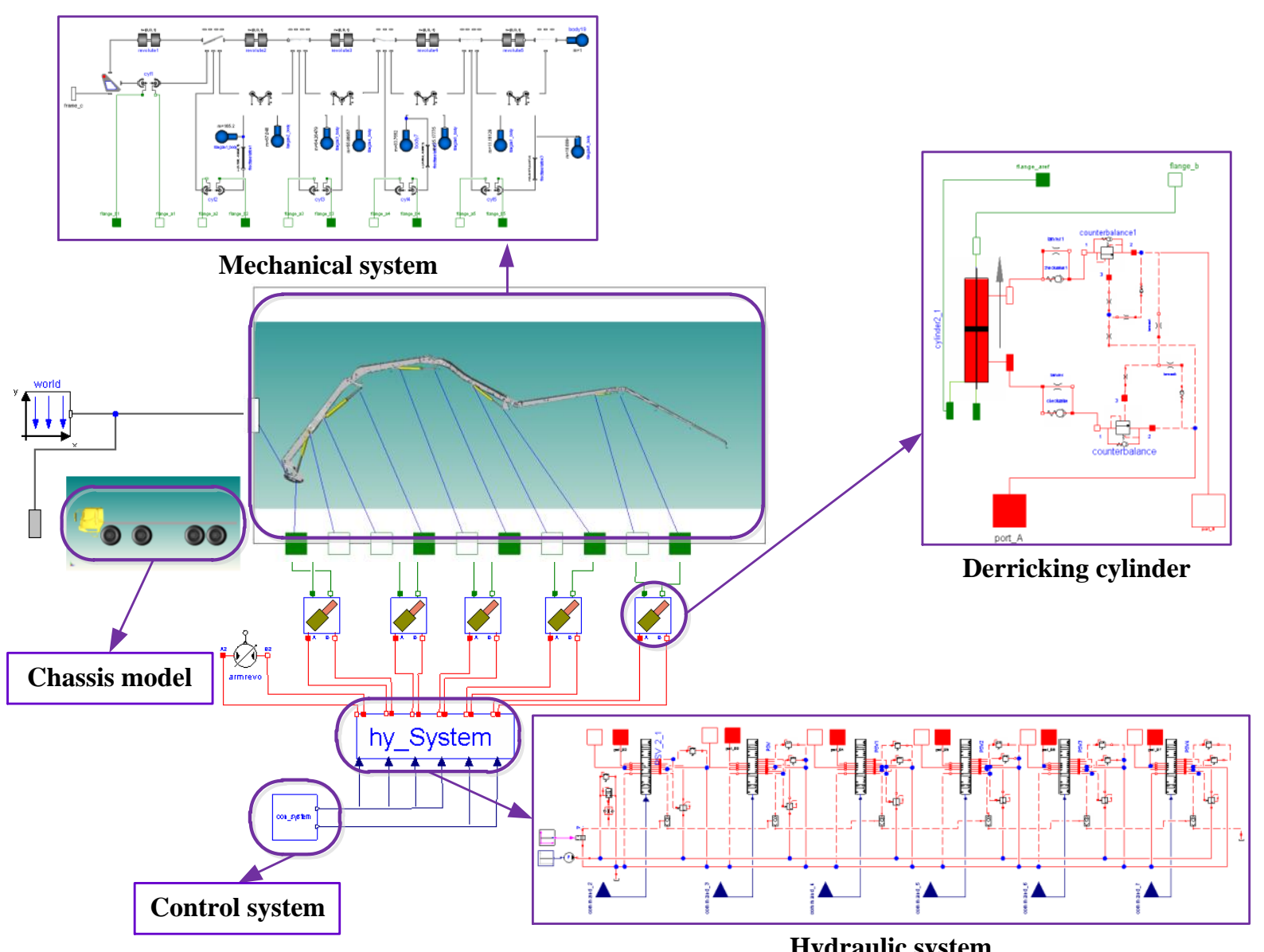

Figure 6: Hierarchical model of the booms system

Base on the system model, the various working conditions can be simulated by modifying the parameters of the control and hydraulic systems, and the work loads of each boom, flow quantity and pressure impact loads in the hydraulic system and the reliabil- ity of control system can also be analyzed. For example, the expanding and folding process of the boom_4 is simulated and the result is shown in Figure 7. 

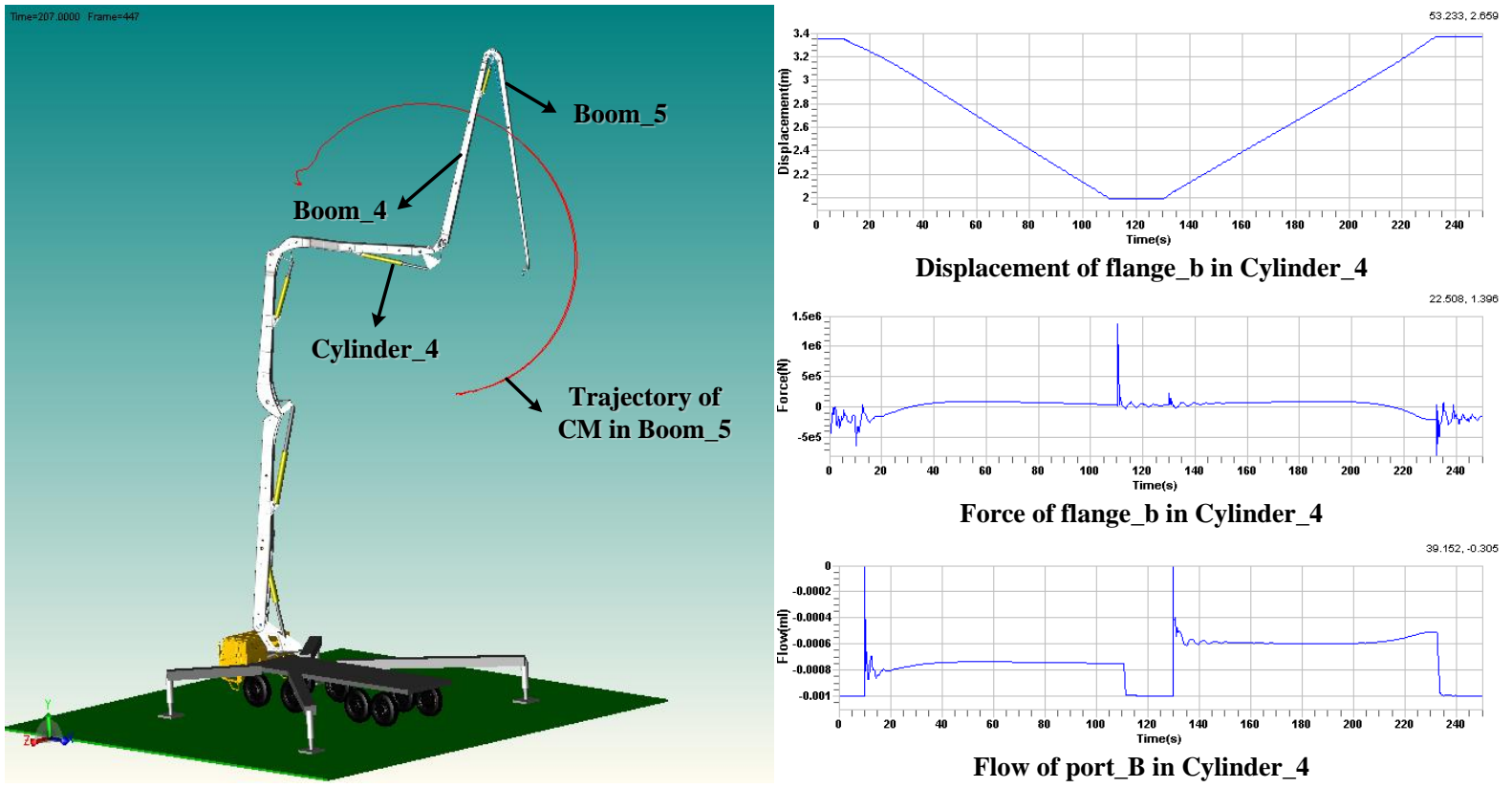

Figure 7: The expanding and holding process of the boom_4

\subsection{Simulation of the Coupled Rigid-flexible Boom System}

There will be a large elastic deformation on each boom in the actual working process. This not only has a great impact on the casting work, but also reduces the safety performance of the system. So it is necessary to take the elastic deformation into consideration for improving the accuracy of the system model.

\subsubsection{Modal Analysis of the Booms}

The modal neutral files of booms are needed to perform the coupled rigid-flexible dynamic analysis. So it's necessary to take modal analysis for each boom.

We use the ANSYS software to compute the modals. The analysis workflow is as follow:

(1) Inputting the material parameters: The elastic modulus, Poisson's ratio, and density are set to FE model. The material properties determine the spring stiffness and damping.

(2) Meshing the model: The solid45 element is selected to mesh the solid geometry. And the mass 21 element is selected to mesh the key points set up on the central axis of the hinge hole.

(3) Configuring the rigid region: The rigid regions are established about the interface node and nodes on the cylinder faces respectively.

(4) Generating the MNF: Run the ADAMS macros, choose the interface nodes, specify the mode order numbers to expand, then generate modal neutral file of each boom.

The finite element models of booms are shown in Figure 8.

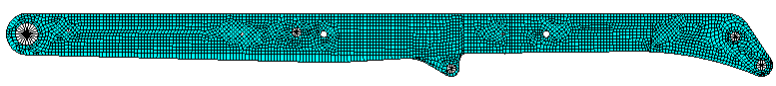

(a) Boom_1

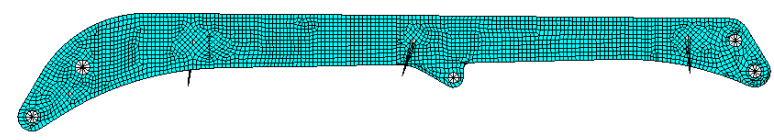

(b) Boom_2

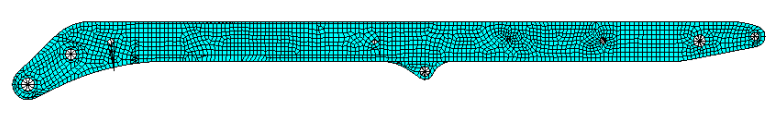

(c) Boom_3

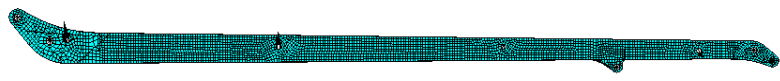

(d) Boom_4

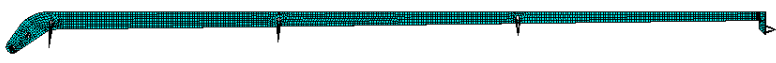

(e) Boom_5

Figure 8: FE model of booms

We set twenty modes to extract for each boom. Then we can get fifty modes in the MNF. The modes from first to sixth are rigid modes, approximately to zero. Because there are five fixed interface points in each boom, and thirty constraint modes of six degrees of freedom are extracted. We choose a sufficient number of modes to represent the boom so that the frequency range is able to deactivate eigenmodes based on the frequency or the energy criterion. Some natural frequencies of the booms are listed in Table 2. 
Table 2: Natural frequency of booms

\begin{tabular}{c|c|c|c|c|c}
\hline $\begin{array}{c}\text { Nat. } \\
\text { Freq. }\end{array}$ & Boom_1 & Boom_2 & Boom_3 & Boom_4 & Boom4 \\
\hline $1-6$ & 0 & 0 & 0 & 0 & 0 \\
\hline 7 & 25.8302 & 35.5842 & 33.0956 & 49.3527 & 8.7492 \\
\hline 8 & 36.3539 & 48.4689 & 49.3527 & 22.1214 & 11.1239 \\
\hline 9 & 52.8219 & 76.9397 & 49.3527 & 53.6334 & 23.7672 \\
\hline 10 & 74.1143 & 89.2075 & 49.3527 & 60.5197 & 30.3335 \\
\hline 11 & 75.6471 & 90.2372 & 49.3527 & 81.8889 & 47.3777 \\
\hline 12 & 81.2592 & 94.7992 & 49.3527 & 85.3635 & 56.8012 \\
\hline$\ldots$ & $\ldots$ & $\ldots$ & $\ldots$ & $\ldots$ & $\ldots$ \\
\hline
\end{tabular}

\subsubsection{Replacement of the FlexibleBody Model}

The rigid parts of booms shown in Figure 6 are replaced by FlexibleBody model with respective parameters, as shown in Figure 9. So the model of the

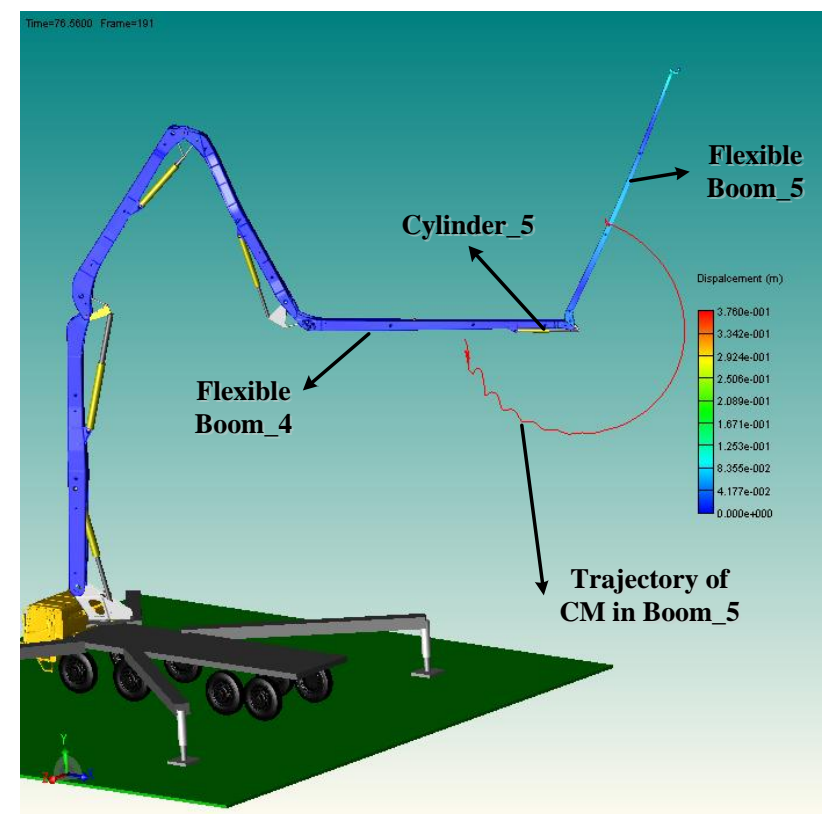

mechanical system is converted from the rigid multibody to the coupled rigid-flexible system.

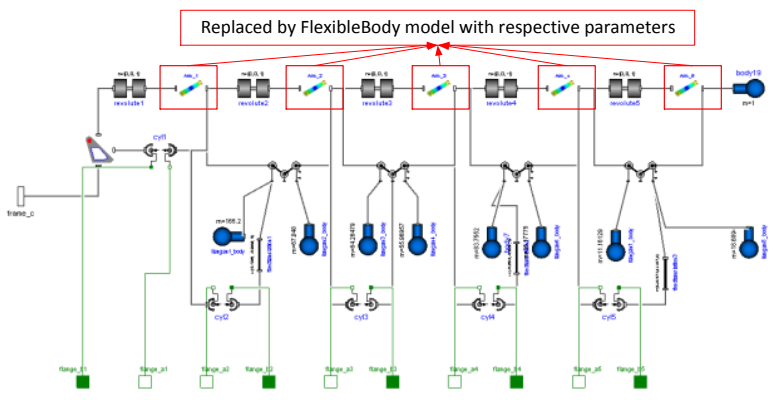

Figure 9: The coupled rigid-flexible mechanical system

\subsubsection{Simulation of Coupled Rigid-flexible Boom System}

The expanding and folding process of boom_5 is simulated, as shown in Figure 10.

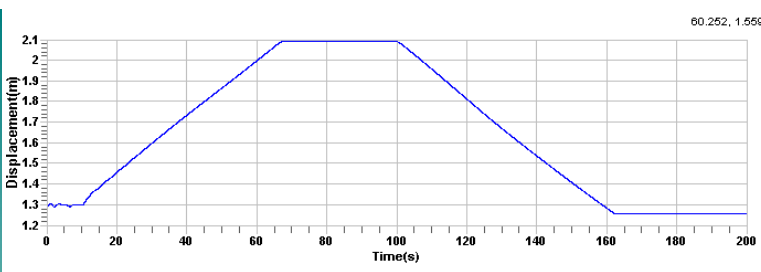

Displacement of flange_b in Cylinder_5

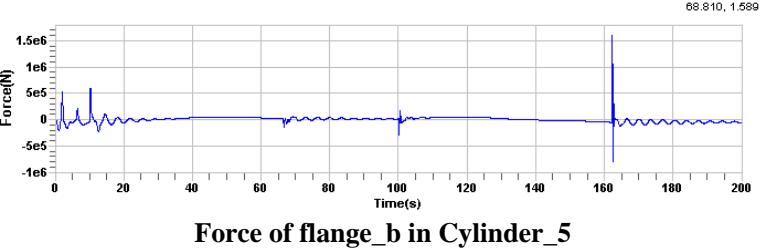

Force of flange_b in Cylinder_5

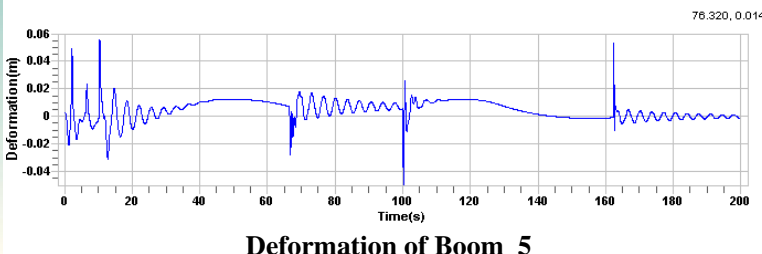

Figure 10: The process of expanding and folding boom_5

The modal coordinates of each boom are show in Figure 11. The modal coordinates q[1] - q[6] are corresponding to $7^{\text {th }}-12^{\text {th }}$ modes respectively. Obviously, the value of modal coordinate $\mathrm{q}[1]$ is the biggest one in each boom. It indicates that the $7^{\text {th }}$ mode contributes most energy to the flexible-body. And the values of other modal coordinates are smaller and smaller, indicating less energy contribution. The variation tendency is complied with the modal superposition theorem and energy criterion ${ }^{[11]}$.

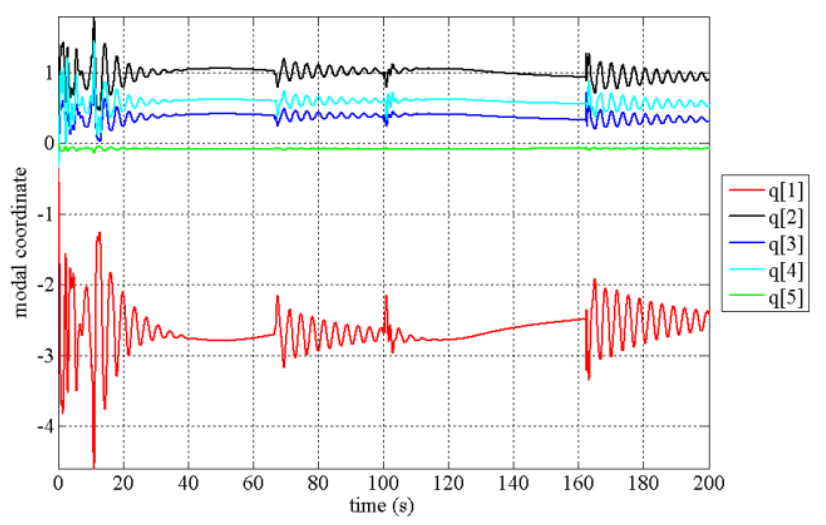

(a) Boom 1 


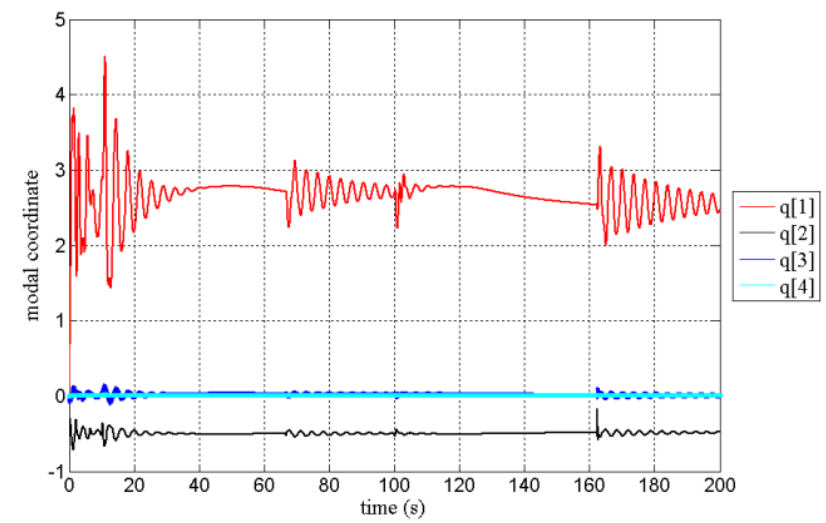

(b) Boom 2

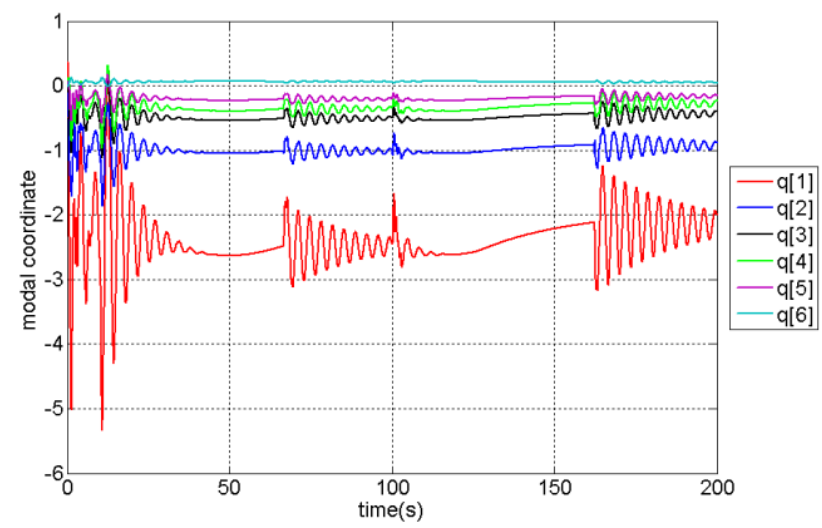

(c) Boom 3

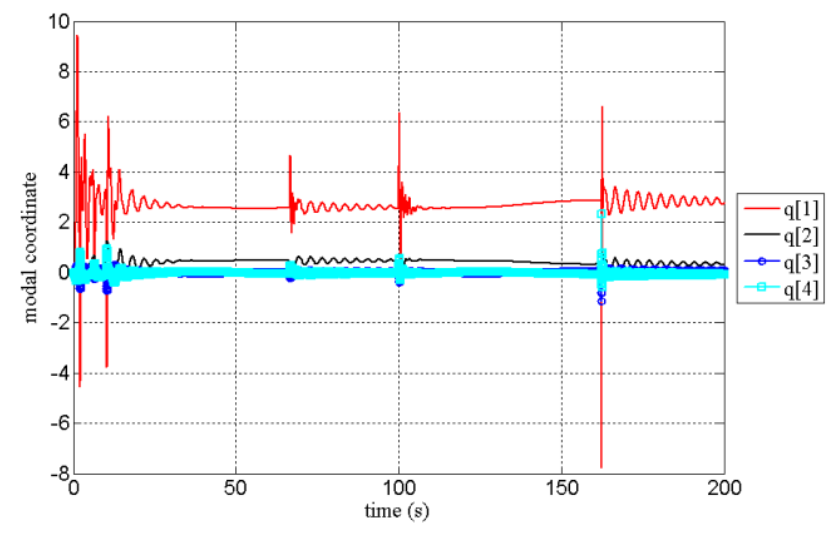

(d) Boom 4

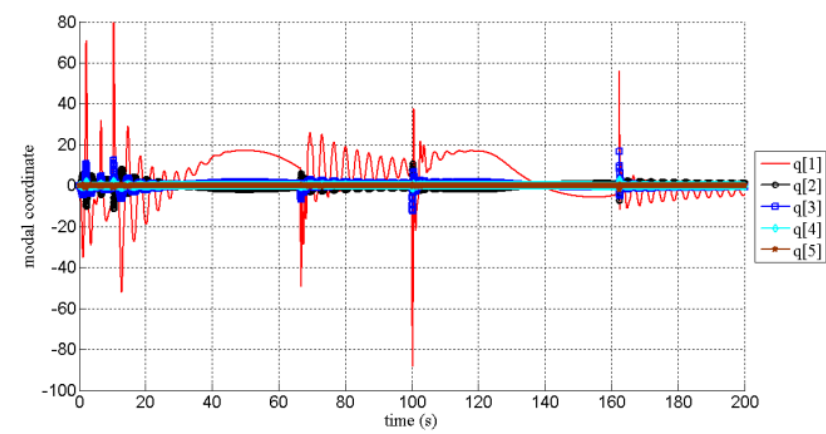

(e) Boom 5

Figure 11: Modal coordinates of booms

With the powerful post-processor in MWorks, the deformation color contour of boom_4 and boom_5 are shown in Figure 12.

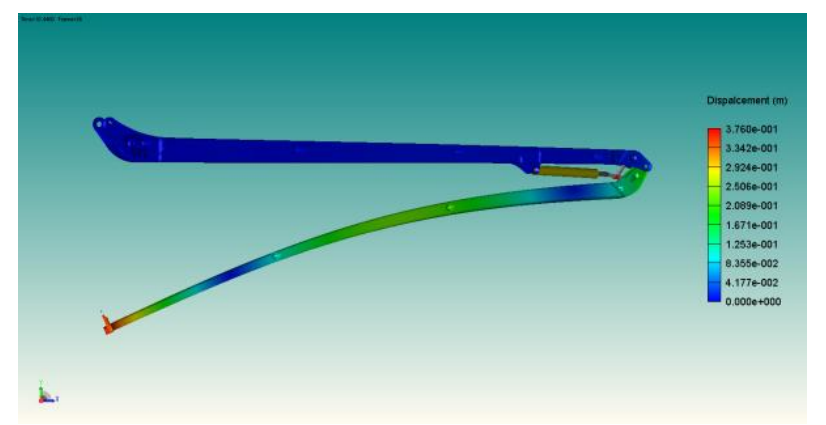

(a) $\mathrm{t}=10.44 \mathrm{~s}$

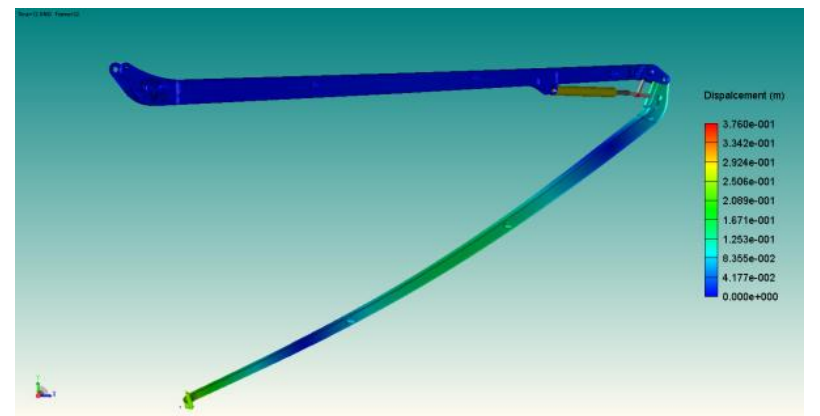

(b) $\mathrm{t}=12.84 \mathrm{~s}$

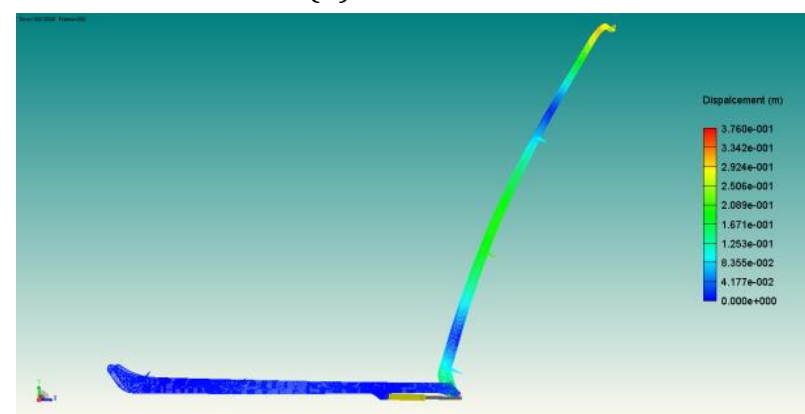

(c) $\mathrm{t}=100.20 \mathrm{~s}$

Figure 12: Nephograms of the deformation in booms

The obvious vibration can be found in simulation result of the coupled rigid-flexible dynamics. And in the actual working process, the vibration does exist in the expanding or the holding process. The impact on hydraulic and control system can be analyzed for the elastic deformation of each boom. So compared with a rigid model, the coupled rigid-flexible model has higher accuracy, and is more close to the actual system.

\subsection{Simulation Comparation between Rigid and Coupled Rigid-flexible Boom System}

\subsubsection{Comparation of Simulation Results}

The Figure 13 shows the simulation results, in comparison coupled rigid-flexible multibody with rigid multibody, of boom_3 boom_5 expanding and holding together. 


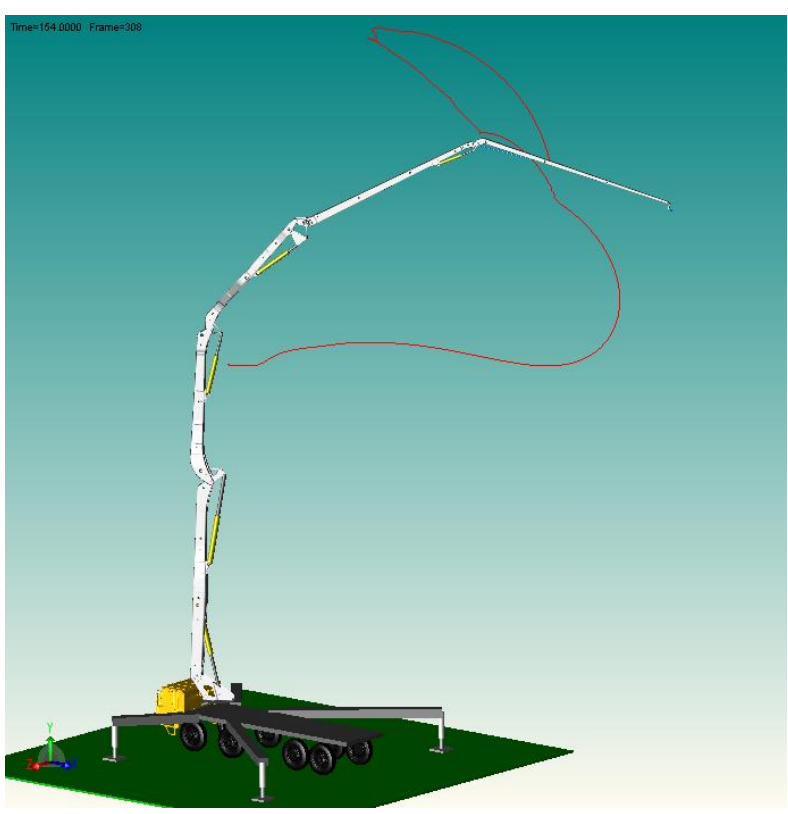

Rigid boom system

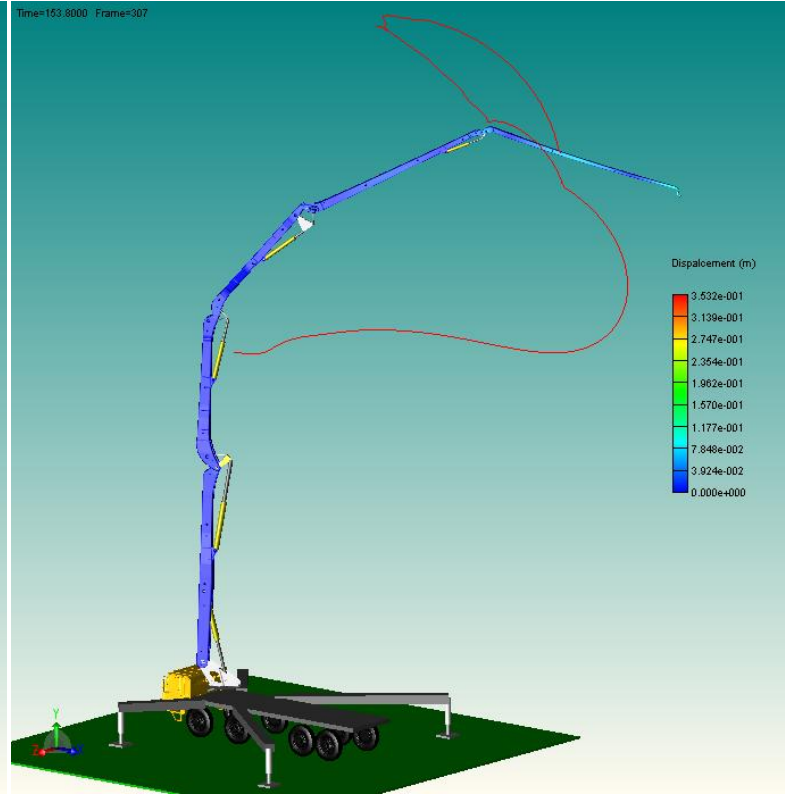

Coupled rigid-flexible boom system

Figure 13: Comparation of rigid system and coupled rigid-flexible system

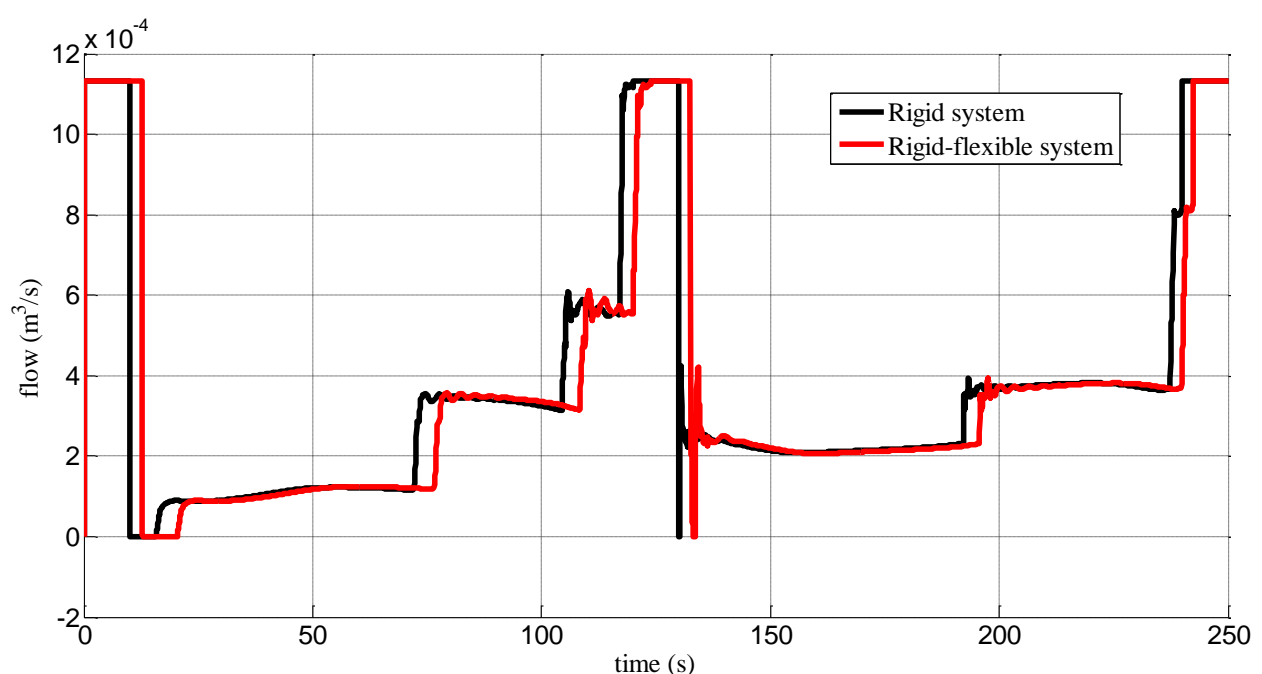

Figure 14: Flow in hydraulic cylinder port_A of boom_4

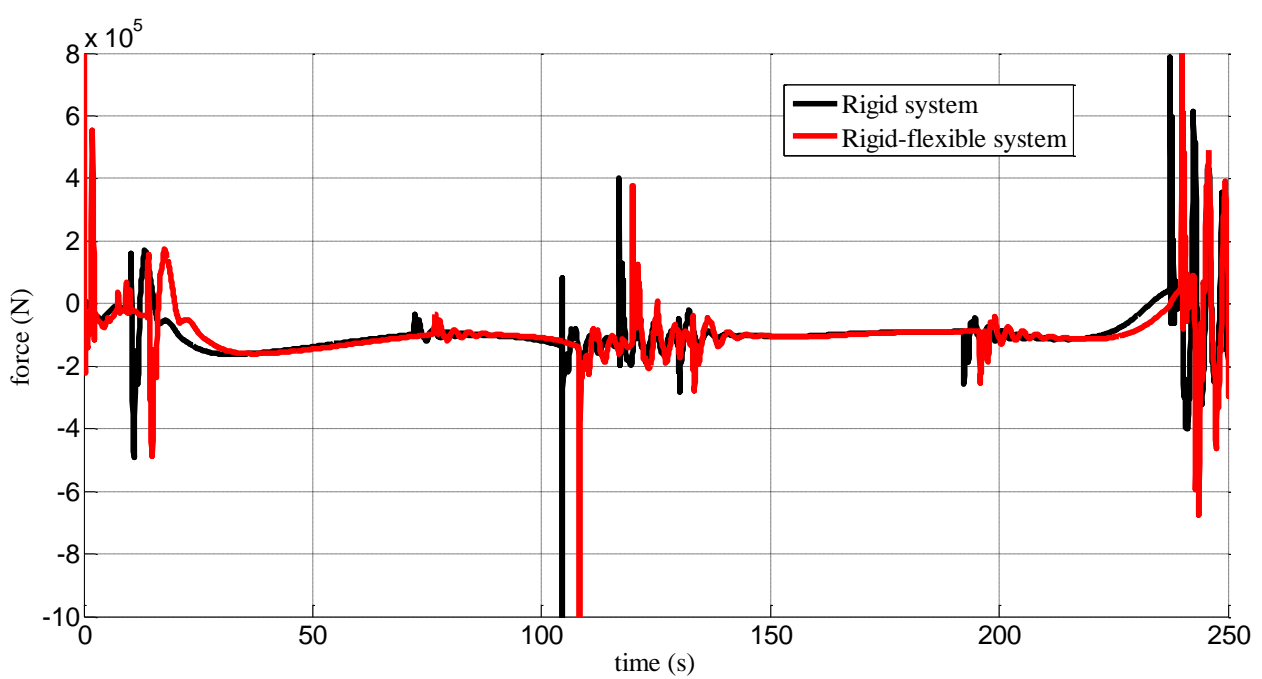

Figure 15: Force in flang_b of boom_4 
Figure 14 shows the flow in hydraulic cylinder port_A of boom_4. Figure 15 shows the force in flange_b of boom_4. There are obvious differences between rigid and the coupled rigid-flexible boom system, especially at the reversing point: the former changes gently, but the latter changes dramatically, which are due to elastic deformation of flexible booms.

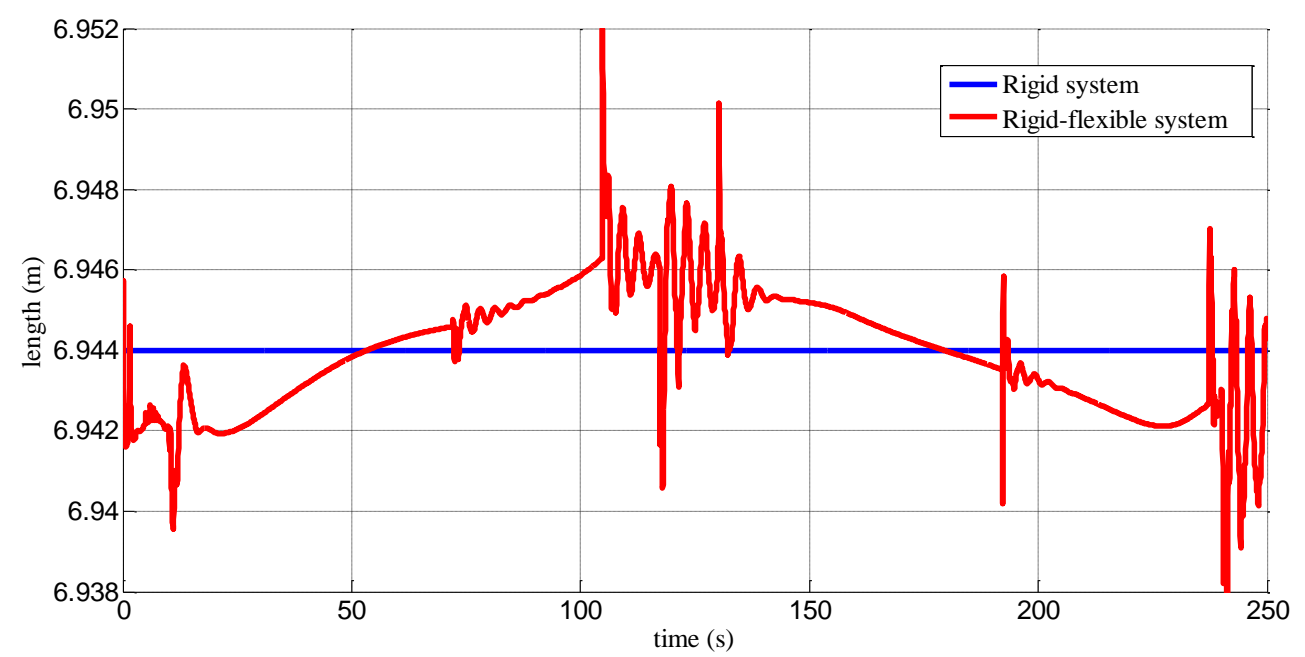

Figure 16: Length of boom_4

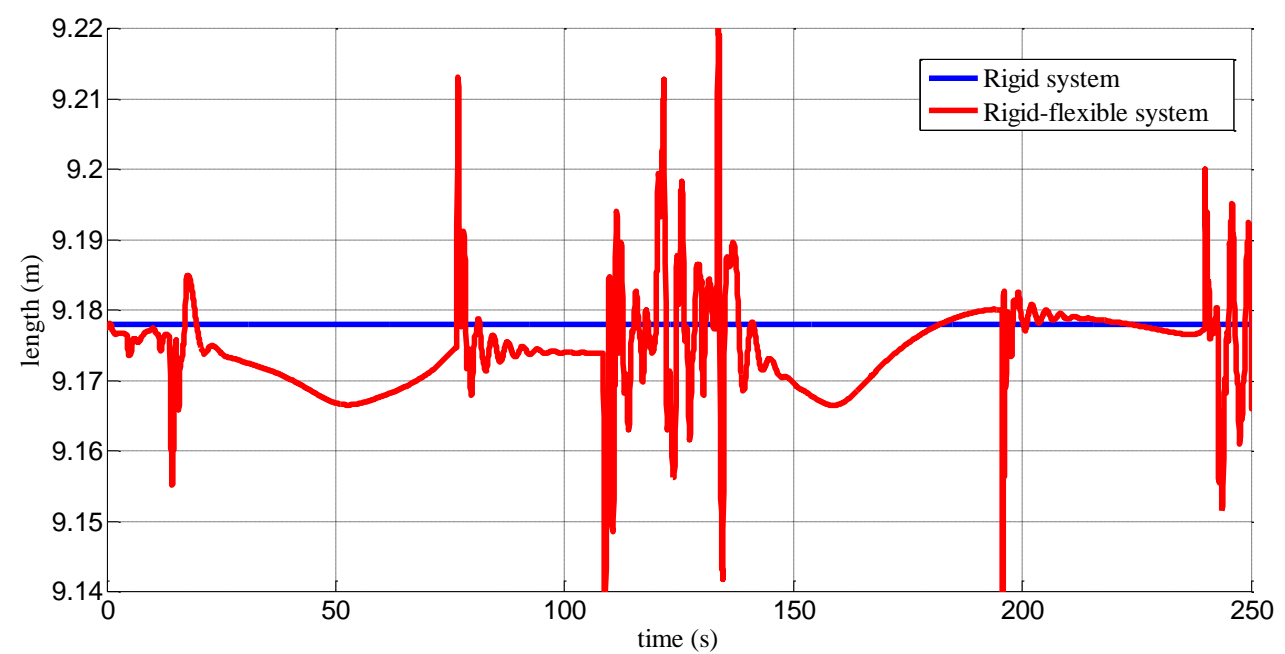

Figure 17: Length of boom_5

Figure 16 and Figure 17 show the length of boom_4 and boom_5 respectively. The length of the rigid boom is a constant value, while the length curve of the flexible boom is shown a relatively evident fluctuation, especially when the motion state changed, deformation peak appears. The maximum force of each hinge point is larger than the rigid body simulation results, when boom system changes the motion state.

\subsubsection{Comparation of Calculation Efficiency}

The finite element method is apt for discretizing the arbitrary complicated geometry. But with gigantic nodes in large scale and complex system, the computation is heavy. FEA is too inefficient for system level modeling and is incapable of analyzing large mo- tion. Moreover, the coupled rigid-flexible system is a strong nonlinear system, especially integrated with hydraulic and control subsystem.

Although introducing great number of variables and equations, component mode synthesis saves time and processing resources by breaking up a single large problem several reduced-order problems via substructuring.

The calculation time for coupled rigid-flexible boom system is more than double for rigid boom system in the test cases. There are several influence factors for calculation time.

(1) The number of nodes. Too many DOFs can mean unacceptably long computation time.

(2) The number of modes. If a mode does not contribute to the response of the flexible component 
during a simulation, it could be disabled to save computation time.

(3) The modal damping coefficients. The bigger modal damping coefficient is helpful to control the integration step by suppressing the resonance response respect to the corresponding natural frequency.

\section{Conclusions}

By introducing the CMS technique and the improved Craig-Bampton method, the FlexibleBody model has been constructed based on the standard MultiBody library in this paper. The boom system of concrete pump truck is modeled and simulated in MWorks, which is composed of a coupled rigid-flexible mechanism, a hydraulic and a control subsystem. Numerical results are compared and discussed with respect to efficiency and accuracy.

The FlexibleBody model can be easily incorporate flexibility into system models. This optional add-on module interface with several commercial finite element applications to accurately define component flexibility, and it has an easy-to-use interface that allows engineers to quickly convert rigid parts to flexible ones.

The simulation process is illustrated by boom system of concrete pump truck applications. However, it can be applied to design any mechanical system such as classical or compliant mechanisms, deformable structures and more general to solve most mechanical dynamics problems.

\section{ACKNOWLEDGEMENTS}

The paper is supported by Major National Science \& Technology Specific Project (No. 2011ZX02403005), The National Basic Research Program of China (973 Program) (No. 2011CB706502).

\section{References}

[1] Andreas Heckmann, Martin Otter, Stefan Dietz, et al. The DLR FlexibleBodies library to model large motions of beams and of flexible bodies exported from finite element programs, The Modelica Association. Modelica 2006: 85-95.

[2] Andreas Heckmann, Stefan Hartweg and Ingo Kaiser. An Annular Plate Model in Arbitrary Lagrangian-Eulerian Description for the
DLR FlexibleBodies Library. Proceedings 8th Modelica Conference, Dresden, Germany, March 20-22, 2011: 121- 132.

[3] O. Wallrapp: Standardization of Flexible Body Modeling in Multibody System Codes, Part 1: Definition of Standard Input Data, Mechanics of Structures and Machines 22(3):283-304, 1994.

[4] P. Koutsovasilis, V. Quarz, M. Beitelschmidt. Standard input data for FEM-MBS coupling: importing alternative model reduction methods into SIMPACK. Mathematical and Computer Modelling of Dynamical Systems, 2009, 15(1): 51-68.

[5] R. Craig, M. Bampton. Coupling of substructures for dynamic analysis. Amer. Inst. Aero. Astro. J. 1968, 6(7): 1313-1319.

[6] S. Rubin. Improved component-mode representation for structural dynamic analysis Amer. Inst. Aero. Astro. J., 13 (8) (1975), pp. 995-1006.

[7] R. R. Craig. Coupling of substructures for dynamic analyses: an overview, in: Structures, Structural Dynamics and Material Conference, 41st AIAA/ASME/ASCE/AHS/ASC, Atlanta, 2000, AIAA-2000-1573.

[8] D. J. Daniel, J. Rixen. A dual Craig-Bampton method for dynamic substructuring. Journal of Computational and Applied Mathematics, 2004, 168(1-2): 383-391.

[9] ADAMS. Theoretical Background. MSC. Software Corporation, 2003: 1-30. http://ti.mb.fh-

osna-

brueck.de/adamshelp/mergedProjects/flex/fle x_gen/flextheory.pdf.

[10] Dimitri Metaxas, Eunyoung Koh. Flexible multibody dynamics and adaptive finite element techniques for model synthesis and estimation. Comput. Methods Appl. Mech. Engrg. 1996, 136: $1-25$.

[11] Takehiko Eguchi. Improvement of component mode synthesis model for vibration analysis of hard disk drives using attachment modes. Microsyst technol, 2007, 13: 10851092.

[12] Frédéric Bourquin. Analysis and comparison of several component mode synthesis methods on one-dimensional domains. Numer. Math. 1990, 58: 11 - 34.

[13] Polarit Apiwattanalunggarn, Steven W. Shaw, Christophe Pierre. Component Mode Synthe- 
sis Using Nonlinear Normal Modes. Nonlinear Dynamics, 2005, 41: 17 - 46.

[14] Ulf Sellgren. Component Mode Synthesis A method for efficient dynamic simulation of complex technical systems. Department of Machine Design, the Royal Institute of Technology, Sweden, 2003: 1-27.

[15] Zhou Fanli, Chen Liping, Wu Yizhong, et al. MWorks: a Modern IDE for Modeling and Simulation of Multi-domain Physical Systems Based on Modelica. The Modelica Association, Modelica 2006: 725-731. 